dynamics. Also, the rotational collisional efficiency is expected to increase as the expansion cools the gas, and therefore the assumption of a constant efficiency is unrealistic.

We have obtained excellent agreement between the generalized Boltzmann flow theory and the experimental distribution measurements for stagnation pressures to approximately 400 Torr. Above this pressure higher terminal rotational temperatures than predicted with $\boldsymbol{\xi}=0.17$ were observed. Such discrepancy in the expansion at high pressure could be due to mechanisms such as molecular clustering which release energy to the flow. In regard to the rotational distributions observed off the central streamline, they remain Maxwellian across a $30^{\circ}$ span from the center and $T_{\text {rot }}$ was constant. It thus appears that, at modest stagnation pressures, molecular free jet flows of pure $\mathrm{N}_{2}$ can now be accurately modeled within a central streamline cone of $60^{\circ}$.

\section{Conclusion}

We have demonstrated an alternative method for studying the rotational evolution of molecular free jet expansions. Our observations show that for $\mathrm{N}_{2}$ the evolution of the highly populated lower rotational levels remains Maxwellian. From the observed signal to noise ratio, that is, our minimum detectable signal, we find less than $0.2 \%$ of the total population can reside in any particular unobserved rotational level. Using this value and the errors in the fit we can conservatively state that better than $95 \%$ of the $\mathrm{N}_{2}$ population is described by a Maxwell-Boltzmann distribution function with a rotational temperature, $T_{\text {rot }}(z)$. For expansions with low stagnation pressure, 400 Torr or less with the nozzles employed in this work, the evolution of $T_{\text {ra }}$ agrees well with a numerical solution of the generalized Boltzmann equation. Further measurements on rotational cooling with a variety of molecules and the connection of these results with the existing studies on translational distributions will provide further evidence useful in mapping the total distribution function of a molecular free jet.

Acknowledgment. This work was supported by the National Science Foundation through Grant No. CHE-8820740. T.L.M. acknowledges Mr. L. K. Randeniya for assistance with the numerical solutions of the generalized Boltzmann flow theory.

\title{
Effects of Chain Length of Surfactants on the Interfacial Tension: Molecular Dynamics Simulations and Experiments
}

\author{
B. Smit, ${ }^{*, \dagger}$ A. G. Schlijper, ${ }^{\ddagger}$ L. A. M. Rupert, ${ }^{\dagger}$ and N. M. van $\mathrm{Os}^{\dagger}$ \\ Koninklijke/Shell-Laboratorium, Amsterdam (Shell Research B.V.), P.O. Box 3003, 1003 AA Amsterdam, \\ The Netherlands, and Koninklijke/Shell Exploratie en Produktie Laboratorium (Shell Research B.V.), \\ P.O. Box 60, 2280 AB Rijswijk, The Netherlands (Received: April 23, 1990)
}

\begin{abstract}
In this letter a simple oil/water/surfactant model is presented. This model allows us to study systematically, using the molecular dynamics technique, the relation between the structure of surfactants and various thermodynamic properties. Specifically, the effect of increasing the hydrophobic tail length on the interfacial tension has been studied. Experimental results on various model surfactants are also presented and compared with molecular dynamics calculations. The experimental data as well as the simulations indicate that increasing the tail length makes the surfactants more effective in decreasing the interfacial tension at low concentration.
\end{abstract}

\section{Introduction}

Amphiphilic compounds (surfactants or blends of surfactants) not only are important for many technological applications, such as tertiary oil recovery or detergency, but are also of interest from a purely theoretical point of view.' In particular, the statistical thermodynamic description of these compounds has received considerable attention recently. ${ }^{2-7}$ In these studies lattice models ${ }^{5-7}$ or (continuum) mean field theories ${ }^{2-4}$ have been used to investigate the relation between the chemical structure of surfactants and their thermodynamic properties.

In principle, it is possible to study an oil/water/surfactant system with all the atomic details by using the molecular dynamics techniques. ${ }^{8-10}$ Although these studies give a wealth of important information on these systems, they at present require too much computer time to calculate the surface properties (e.g., surface tension) to a sufficiently accurate degree to allow a systematic study. In this letter we present an oil/water/surfactant model that is simplified to such an extent that some aspects of the relation between surfactant structure and the interfacial properties can be studied. The model still retains the essential features, as is demonstrated by its validation through comparison with new experimental data for a sequence of model compounds.

\footnotetext{
Koninklijke/Shell-Laboratorium, Amsterdam

† Koninklijke/Shell Exploratie en Produktie Laboratorium.
}

\section{Model and Computational Details}

In our model, which is based on an early model of Telo da Gama and Gubbins, ${ }^{11,12}$ we assume the existence of two types of particles: "oillike" and "waterlike". These two types of particles are used to model three types of molecules, namely, oil molecules, water molecules, and surfactant molecules. An oil molecule consists of a single oillike particle, and a water molecule consists of a single waterlike particle. A surfactant molecule is made up of one waterlike particle and one or more oillike particles; these are joined

(1) Mittal, K. L., Lindman, B., Eds. Surfactants in Solution; Plenum Press: New York, 1984; Vols. 1-3

(2) Gruen, D. W. R. J. Phys. Chem. 1985, 89, 146

(3) Szleifer, I. Statistical Thermodynamics of Amphiphilic Aggregates. Ph.D. Thesis, Hebrew University, Jerusalem, 1988

(4) Szleifer, I.; Ben-Shaul, A.; Gelbart, W. M. J. Chem. Phys. 1987, 86, 7094-7110.

(5) Leermakers, F.; Scheutjens, J. M. H. M. J. Chem. Phys. 1988, 89, 6912-6924

(6) Leermakers, F. Statistical Thermodynamics of Association Colloids. Ph.D. Thesis, Landbouwhogeschool Wageningen, The Netherlands, 1988.

(7) Dill, K. A.; Cantor, R. S. Macromolecules 1984, 17, 380.

(8) van der Ploeg, P.; Berendsen, H. J. C. Mol. Phys. 1983, 49, 233.

(9) Lindse, P. J. J. Chem. Phys. 1987, 86, 4177.

(10) Carpenter, I. L.; Hehre, W. J. J. Phys. Chem. 1990, 94, 531

(11) Telo da Gama, M. M.; Gubbins, K. E. Mol. Phys. 1986, 59, 227.

(12) Smit, B. Phys, Rev. A 1988, 37, 3431-3433. 
(a) SIMPLE OIL/WATER/SURFACTANT MODEL

"WATER-LIKE" PARTICLE.

"OIL-LIKE"PARTICLE

SURFACTANT PARTICLE

(b) EXTENDING THE HYOROPHOBIC TAIL OF THE SURFACTANT

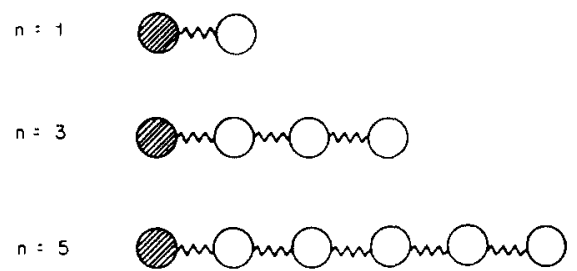

Figure 1. Schematic drawing of the model surfactant/water/oil mixture: (a) simple model; (b) extending the hydrophobic tail of the surfactant.

together by harmonic potentials (Hookean springs; see also Figure 1a).

The two types of particles interact with truncated Lennard-Jones potentials with energy parameter $\epsilon_{i j}$, distance parameter $\sigma_{i j}$, and the cutoff radius $R_{i j}^{\mathrm{c}}$ :

$$
\begin{aligned}
& V_{i j}=\left\{\begin{array}{cc}
\phi_{i j}(r)-\phi_{i j}\left(R_{i j}^{\mathrm{c}}\right) & r \leq R^{\mathrm{c}} \\
0 & r>R_{i j}^{c}
\end{array}\right. \\
& \phi_{i j}(r)=4 \epsilon_{i j}\left[\left(\sigma_{i j} / r\right)^{12}-\left(\sigma_{i j} / r\right)^{6}\right]
\end{aligned}
$$

where $i$ and $j$ indicate the type of atom ("waterlike" or oillike") and $r$ is the distance between the atoms.

Of course, use of a Lennard-Jones type potential represents a drastic simplification. Therefore, we have not tried to optimize the Lennard-Jones parameters for the various interactions. We have assumed that for all interactions $\epsilon_{i j}=\epsilon$ and $\sigma_{i j}=\sigma$. To make the oil-oil and water-water interactions different from the water-oil interactions, the truncation of the potential $\left(R_{i j}^{c}\right)$ is made depending on the type of interactions. For the water-water and oil-oil interactions the potential is truncated at $R^{c}{ }_{i j}=2.5 \sigma$ and for the oil-water interaction at $R_{i j}^{c}=2^{1 / 6} \sigma$, which makes the latter interaction completely repulsive.

These definitions for the interactions make the surfactant behave as an amphiphilic molecule: one side likes the water and dislikes the oil, the other side likes the oil and dislikes the water. With this model it is very simple to mimic changes in the chemical structure. For example, addition of oillike particles to the tail allows us to study the influence of increasing the "tail length" of a surfactant (see Figure 1b).

In our simulations we have used a system of two layers of 256 waterlike particles and 512 oillike particles placed on an foc lattice at the center of a periodic box of size $7.15 \sigma \times 7.15 \sigma \times 21.45 \sigma$. The density was $\rho=0.7 \sigma^{-3}$, and the temperature was kept constant at $T=1.0 \epsilon / k_{\mathrm{B}}$. The total number of particles was kept constant by replacing oil and water particles by surfactants.

After the equilibration the components of the pressure tensor and density profiles were calculated every tenth time step. The interfacial tension was calculated by integrating the difference of the normal and tangential components of the pressure tensor over the interface. ${ }^{12}$ The accuracy of our simulations was estimated by dividing the simulation into subruns and calculating the standard deviations from block averages. Each total simulation took at least $1.5 \times 10^{6}$ time steps (time step $\Delta t=0.005 \tau_{0}$, where $\tau_{0}$ is the reduced unit of time $\left.\tau_{0}=\sigma(m / \epsilon)^{1 / 2}\right)$.

\section{Results and Discussion}

The effect of the chain length of surfactants has been studied experimentally for sodium $p$ - $(x$-alkyl) benzenesulfonates, with chain

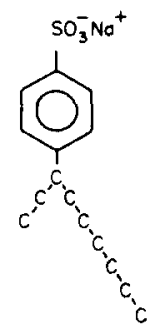

$\mathrm{C}_{\mathrm{g}}$

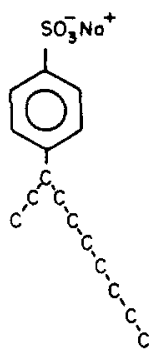

$\mathrm{C}_{70}$

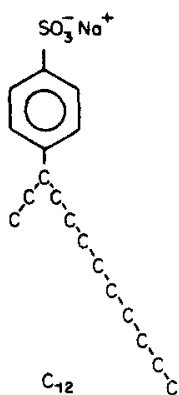

Figure 2. Schematic structure of the sodium $p$ - $(x$-alkyl)benzenesulfonates.

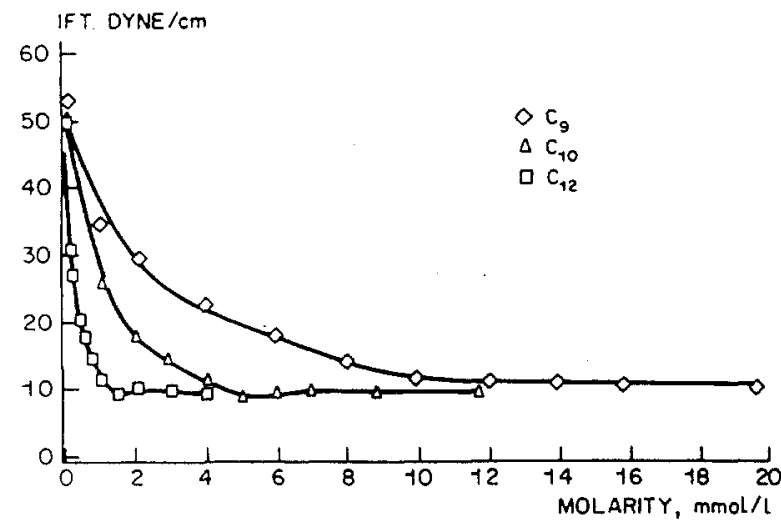

Figure 3. Interfacial tension as a function of the surfactant concentration for various sodium $p$ - $\left(x\right.$-alkyl) benzenesulfonates at $25^{\circ} \mathrm{C}$.

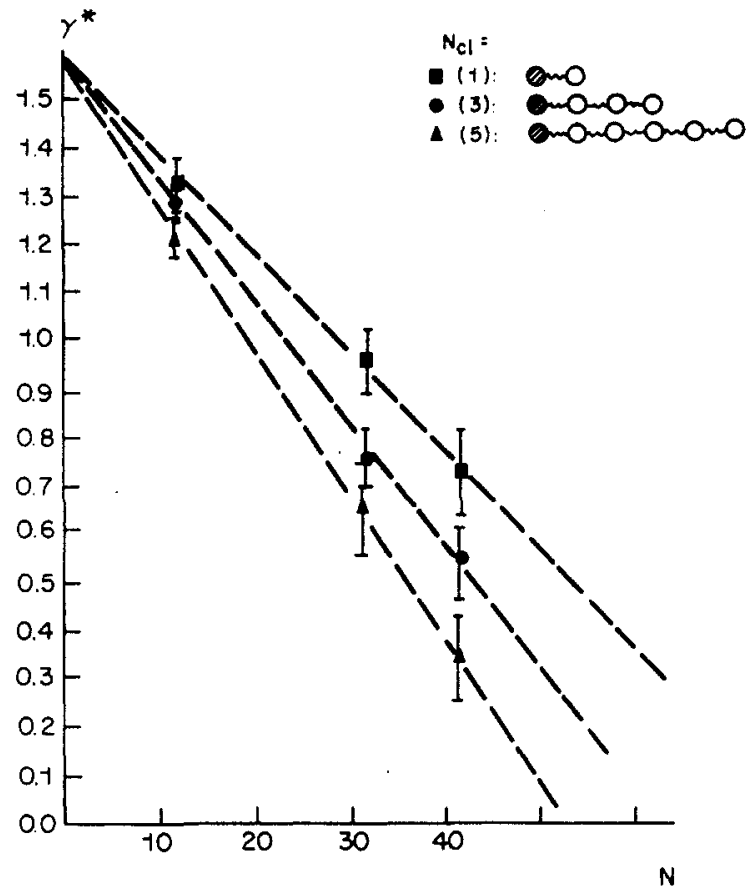

Figure 4. Reduced interfacial tension $\gamma^{*}=\gamma \sigma^{2} \epsilon^{-1}$ as a function of the total number of surfactants $(N)$ for various chain lengths $\left(N_{\mathrm{cl}}\right)$ as obtained from the molecular dynamics simulations.

lengths from $\mathrm{C}_{9}$ to $\mathrm{C}_{12}$. These compounds are described in detail elsewhere ${ }^{13,14}$ and are shown schematically in Figure 2. For these compounds the interfacial tension of a decane/water interface was measured as a function of the surfactant concentration by using a semiautomatic Lauda tensiometer that employs the du Nouy ring method.

(13) van Os, N. M.; Daane, G. J. R.; Bolsman, T. A. B. M. J. Colloid Interface Sci. 1987, $115,402$.

(14) van Os, N. M.; Daane, G. J. R.; Bolsman, T. A. B. M. J. Colloid Interface Sci. 1988, 123, 267. 


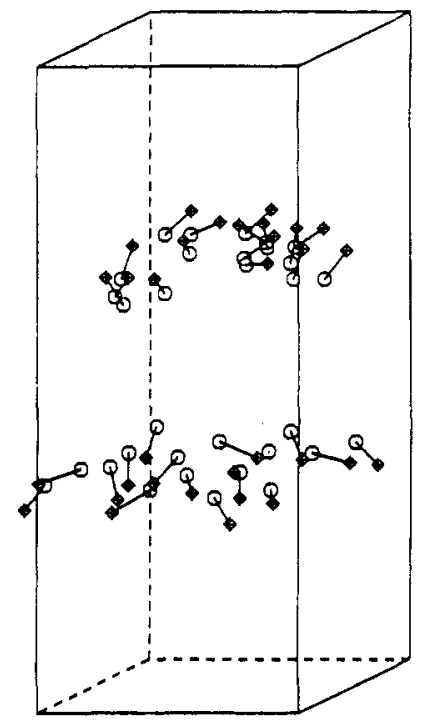

50

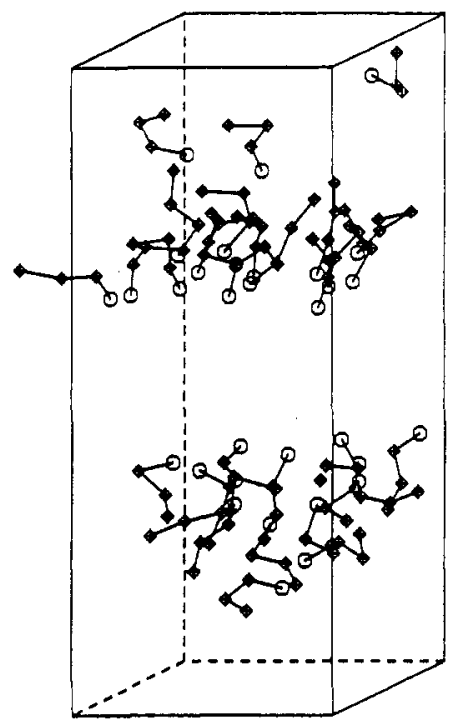

$5 b$

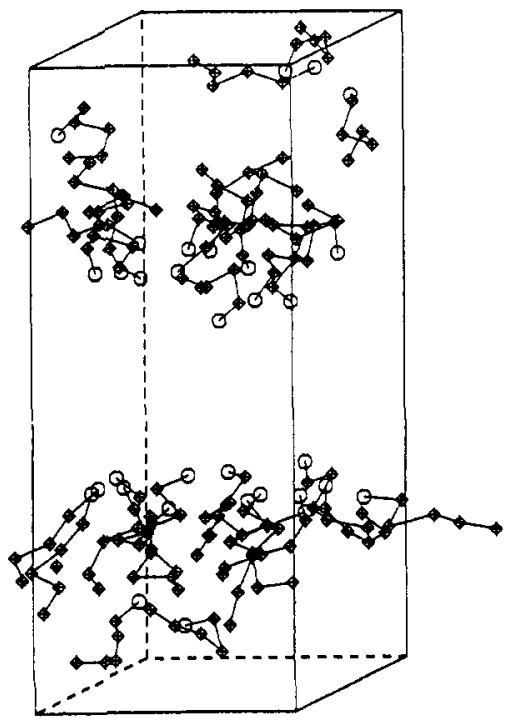

$5 c$

Figure 5. Snapshots of the positions of the surfactants (note that for clarity the positions of the oil and water particles are not drawn). A head group is depicted by $O$ and a tail segment is depicted by $\bullet$. The total number of amphiphiles is 32 ; in (a) the number of tail segments is 1 , in (b) 3 , and in (c) 5 .

The effect of increasing the chain length of surfactants on the interfacial tension is given in Figure 3 for the experimental results and in Figure 4 for the molecular dynamics calculations. The experimental data show that longer tails are more effective at low concentration, i.e., one needs less surfactant to achieve the same reduction of the surface tension.

In Figure 5 snapshots of the surfactant particles are given. This figure shows that the surfactants are preferentially located at the interface. It is interesting to note that the ordering of the surfactants at the interface is less ideal than textbooks suggest. This is in agreement with the single-chain mean-field calculations of Szleifer et al..$^{15}$ and the molecular dynamics simulations on bilayers by van der Ploeg and Berendsen.

Because of the simplifications in our model a direct quantitative comparison with the experimental data is not possible. However, it is of interest to compare the results qualitatively. The simulations demonstrate that the surface tension decreases linearly as the number of surfactants increases. Because of our relatively small system we could not extend our simulation to a larger number of surfactants to see whether our model predicts the formation of micelles. In the experimental curves a characteristic break in the interfacial tension curves is observed at high concentrations of surfactants. This is due to the formation of micelles in the water phase. 5081 .

(15) Szleifer, I.; Ben-Shaul, A.; Gelbart, W. M. J. Phys. Chem. 1990, 94,
Comparison of the simulation results with the experimental data at low concentration of surfactants shows that the model calculations predict the relationship between the tail length and the interfacial tension correctly, namely, increasing the tail length makes the surfactants more effective. It is interesting to note that these results for the interfacial tension are in good agreement with the calculations of Szleifer et al. ${ }^{15}$ They have used a mean-field theory to calculate the lateral pressure as a function of the head-group area for surfactants with different chain lengths. If we assume that the head-group area is approximately constant for all chain lengths, ${ }^{16}$ these calculations also demonstrate that as the chain length increases the lateral pressure increases and therefore the surface tension decreases.

In summary, in this letter we have demonstrated that with a very simple oil/water/surfactant model aspects of the relation between the structure of a surfactant and its surface activity can be studied. The full results of our simulations will be published elsewhere.

Acknowledgment. B.S. thanks I. Szleifer for sending him preprints prior to publication.

(16) Comparison of the density profiles obtained from the simulations shows that at low concentration the average head-group area is equal for all chain lengths, at higher concentration the head-group area is slightly smaller for the longer chains. 